\title{
Optimization and Validation of a Fingerprint about Hypericum perforatum L. Extracts by Plackett-Burman Randomization Method
}

\author{
Shu Jie Shen ${ }^{1}$, Yan Ru Liu ${ }^{1,2}$, Chun Hua Guo ${ }^{1,3}$, Rong Qing Huang ${ }^{1 *}$, Jian Yun Yang ${ }^{1}$ and Bing Kun Xiao ${ }^{1}$
}

${ }^{1}$ Beijing Institute of Radiation Medicine, No. 27 Taiping Road, Haidian District, Beijing 100850, PR China

2Shanxi Collaborative Innovation Center of Chinese Medicinal Resources Industrialization, Shaanxi University of Chinese Medicine, No.1 Middle Weiyang Road, Qindu District, Xianyang, Shaanxi, PR China

3Jiangxi University of Traditional Chinese Medicine, No.18, Yunwan Road, Wanli District, Nanchang 330004, PR China

"Corresponding author: Huang R-Q, Beijing Institute of Radiation Medicine, NO. 27 Taiping Road, Haidian District, Beijing 100850, PR China, Tel: 86 13681493410; Email: rqhuang@aliyun.com

Rec date: Nov 04, 2014, Acc date: Jan 07, 2015, Pub date: Jan 09, 2015

Copyright: (c) 2015 Shen S, et al. This is an open-access article distributed under the terms of the Creative Commons Attribution License, which permits unrestricted use, distribution, and reproduction in any medium, provided the original author and source are credited.

\begin{abstract}
Hypericum perforatum L. (HPL) has been used as a beneficial herb on menopause related syndromes for many years by inhibiting lipid oxidation. Hypericum perforatum L. extract contain flavonoids and phenolic acids, but it was not a method to separate and identify flavonoids which was based on design of experiment. Moreover, upper complicated HPLC method might cause undesirable effects on feasibility and repeatability in fingerprint technology. It was necessity to establish a generally and systematized way which could develop a reliable fingerprint technology for Chinese medicine. Plackett-Burman design method was a conventional tool for variables randomization aiming at optimization. Our study was based on this optimized design of experiment about ethanol extract from Hypericum perforatum L. and established the corresponding method for separation and identification of flavonoids. With this Design-Expert software, the results showed HCRF have significant effect on variables. From One-factor plots for main effect of HCRF, $254 \mathrm{~nm}$ wavelength measurement have a significant influenced on the HCRF, and have provided the optimized parameters.
\end{abstract}

Keywords: Plackett-Burman (PB) design; Flavonoids; Fingerprint technology

\section{Introduction}

Menopausal syndrome was one of the important factors, which affects the interference middle-aged and old women's life quality for many years. Hypericum perforatum L. has been used for the treatment of antidepressant and antioxidant widely. Plackett-Burman design method was a conventional tool for variables randomization. Such diversity could be within the same species. For that differentiated strains might have some minor or major differences $[1,2]$. This design method could enable randomizing different variables aiming to get the best conditions where each variable coordinates with other variables to give the best expected results [3]. Simple tools could be used to conduct complicated target if the correct variables have been used or if successful alternative variables are used as well. The main pharmacological activity of Hypericum perforatum L. was flavonoids. Flavonoids actively ingredients could remove ABTS $\bullet+(2,2$ '-azino-bis(3-ethylbenzthiazoline-6-sulfonic acid) on free radical) radical activity [4]. While the current study had not established the appropriate methods to separate and to identify about flavonoids on optimal design experiments (DOE, Design of Experiment) on the ethanol extract of Hypericum perforatum L. In addition, HPLC method might affect the feasibility and the reproducibility of fingerprints. Therefore, it is necessary to establish a reliable and easily systematic approach of Hypericum perforatum L. fingerprints. We choose five common and representative Hypericum perforatum L. flavonoids (rutin, hyperoside, quercitrin, hypericin and quercetin) based on the Plackett-Burman randomization method. This study was based on the optimized design of ethanol extract from Hypericum perforatum L. and aimed at established the corresponding method for separation and identification of flavonoids.

\section{Materials and Methods}

\section{Materials}

Standard extracts rutin (purityN97\%), hyperoside (purityN98\%), quercitrin (purityN98\%) and quercetin (purityN98\%) from HPL were obtained commercially from Must Biotechnology CO., LTD. (Chengdu, China).

\section{Regents}

Methanol (Fisher, Fair Lawn, NJ, USA) and formic acid (Fluka, Buchs, Switzerland) were of HPLC grade. The pure-distilled water used was Watsons pure distilled water.

\section{Screening design and main effect plot}

Hypericum perforatum L. and reference substance solution preparation: The standard sample of rutin, Hyperoside, quercitrin and quercetin were weighed accurately, and dissolved in methanol to prepare the solution of compounds into $0.1 \mathrm{mg} / \mathrm{mL}$, respectively. The same operation of Hypericum perforatum $\mathrm{L}$. from alcohol extract into $2 \mathrm{mg} / \mathrm{ml}$. 
Page 2 of 5

\section{The design of Chromatography elution system impact factor}

This study was based on the formula of liquid chromatography, the elution dynamics system and the thermodynamics factors could be optimized design. In the pre-experiment, acetonitrile water system has been proved preferable results of separation condition. And specific concentrated formic acid for $\mathrm{pH}$ of mobile phase system has been selected as a modifier. Then other variables such as oven temperature was depend on experimental condition.

\section{Statistical analysis}

Design-Expert statistical software (Version8.0.6, Minneapolis, MN) was used to design and to analysis. In this study, the experimental P-B design $(\mathrm{N}=12)$ was used to identify the main factors affecting the responses among numerous variables [5]. Multifactor (such as the detection wavelength, column temperature, gradient time, sample volume, flow rate, concentration of acid, initial proportion of acetonitrile) were analysed of variance (ANOVA) after the HPLC analysis process. This experimental was paralleled operation three times. In this study, an experimental Box-Behnken response surface design was built to optimize the main factor which was affects the response among variables optimal parameters setting.

\section{Optimization of a liquid chromatography factor}

The ratio of liquid phase organic solvent was an important factor about the retention time. Due to the methanol has interacts with analyte hydrogen bond and stronger polarity than acetonitrile. So we selected acetonitrile as organic phase. Besides, column temperature was another parameter that affecting retention time of polar compound. In facts, with the increasing temperature and elevating diffusion, coefficient could generate narrow peaks and higher resolution. Taking the life-span of the column into account, the column temperature range was set in $25 \sim 40^{\circ} \mathrm{C}$. So the ODS column and the mobile phase of acetonitrile-water were selected as a separate system. Meanwhile, a low concentration of formic acid was added to suppress the ionization of flavonoids and other carboxylic acids.

\section{Plackett-Burman (PB) design of experiment}

To optimize the controllable factors, including separates of column temperature, detection wavelength, injection volume, gradient elution parameters and initial concentration of organic solvent, PlackettBurman (PB) test design method was applied, which allows the number of possible factors to be minimized with an efficiency option. Our study adopts four corresponding factors $\Sigma$ Rs, $\mathrm{r}^{\star}\left(\mathrm{x} 0^{-3}\right)$ and HCRF to be evaluated for the quality of the fingerprint from many aspects (as seen table 1). Besides, four corresponding factors were imported in Desigtn-Expert statistical software (Version8.0.6, Minneapolis, MN) which was based on the Plackett-Burman (PB) pilot program. It is shows that the relationship between variables and HCRF regression equation have statistically significant $(\mathrm{p}=0.0053)$. As a result, the equation of HCRF could evaluate the quality of fingerprints.

$\mathrm{HCRF}=1,000,000 \mathrm{n}+100,00 \mathrm{R}_{\min }+\left(\mathrm{t}_{\mathrm{m}}-\mathrm{t}_{1}\right)$ (formula 1 )

The effect on HCRF had determined with the significant $p$ values. By the analysis of variance, learning that the statistically significant have influenced on variable factors which contains: detection wavelength, initial organic phase proportion, injection volume and velocity of flow $(p=0.0005, p=0.0005, p=0.032, p=0.0444)$, while other factors had no effect on HCRF $(\mathrm{P}>0.05)$ (Figure 1).

\begin{tabular}{|l|l|l|l|}
\hline$\sum R s$ & $r^{*} \times 10^{-3}$ & $\Phi$ & HCRF \\
\hline 21.41 & 129.6382 & 56.01743 & 27066001 \\
\hline 46.52 & 82.33626 & 35.86686 & 10187007 \\
\hline 39.29 & 147.7798 & 38.9847 & 15066009 \\
\hline 123.36 & 215.547 & 47.86575 & 5483008 \\
\hline 90.36 & 0.098556 & 467.4022 & 56060001 \\
\hline 57.02 & 16.79956 & 42.18741 & 10160008 \\
\hline 10.33 & 703.5344 & 49.17757 & 58047001 \\
\hline 11.61 & 744.7321 & 56.39541 & 50043003 \\
\hline 8.27 & 547.1393 & 52.85101 & 77055000 \\
\hline 94.35 & 50.21223 & 47.18402 & 7154009 \\
\hline 104.26 & 74.18386 & 73.93493 & 10176007 \\
\hline 20.35 & 137.4934 & 55.45506 & 25051005 \\
\hline
\end{tabular}

Table 1: Table response results.

From One-factor plots for main effect of HRCF, we found that the $254 \mathrm{~nm}$ wavelength measurement had effect on the HCRF distinctively. Column temperature and injection volume had elevated the spectrum of HCRF, but the longer gradient would cause the higher initial ratio organic phase. Greater acid concentration and faster flow rate were still left much to be desired in analysis.

\section{Box-Behnken response surface analysis}

The Box-Behnken response surface design was applied to find more about optimized significant factors. Hoping to find the maximum setting of HCRF, the experimental for Box-Behnken was established with four factors and three levels design.

The equation was: $\mathrm{Ey}=\mathrm{b} 0+\mathrm{b} 1 \mathrm{z} 1+\ldots+\mathrm{b} 4 \mathrm{z} 4+\mathrm{b} 11 \mathrm{z} 12+\ldots+\mathrm{b} 11 \mathrm{z} 12+$ b12z1z2+...b34z3z4

From the analysis of variance, the corresponding $p$ value $=0.1098$. It indicated that the equation had small proportion in the actual fitting of non-normal errors and had intimated correspond with factors. Response surface plots shows the inject volume and the detective wavelength for (a) and (b) at $254 \mathrm{~nm}$ and $20 \mathrm{ul}$, respectively; and the detective wavelength and initial concentration of acetonitrile for (c) and (d) at $254 \mathrm{~nm}$ and 15\%, respectively (Figure 2).

As shown in the picture, with the same detection of wavelength, the detection wavelength had better effects on the surface than the increasing sample volume (Figure $2 \mathrm{a}$ and $2 \mathrm{~b}$ ). With the increasing initial organic phase, the response surface decreased gradually, but the flow velocity had no effect on it (Figure $2 \mathrm{c}$ and $2 \mathrm{~d}$ ). Then, the lower organic could contribute to separate polar components in Hypericum perforatum L. (mainly flavonoids). In other words, the detection wavelength and the initial ratio of organic phase had no interacting effect on the response factors.

With the maximum response factor from the equation, we obtain the best optimal parameters. After three repeated tests to verify the results of factor analysis, the result shows: Column: Diamonsil C18 (250 mm x $4.6 \mathrm{~mm}$ I.D., $5 \mu \mathrm{m}$.), Detection wavelength: $254 \mathrm{~nm}$, 
Citation: Shen S, Liu YR, Guo CH, Huang RQ, Yang JY, et al. (2014) Optimization and Validation of a Fingerprint about Hypericum perforatum

column temperature: $40^{\circ} \mathrm{C}$, gradient time: 25 min, sample volume: 25

$\mu$ l, Flow rate: $0.8 \mathrm{ml} / \mathrm{min}$, the initial ratio of acetonitrile: $15 \%$, the acid concentration: $0.05 \%$.
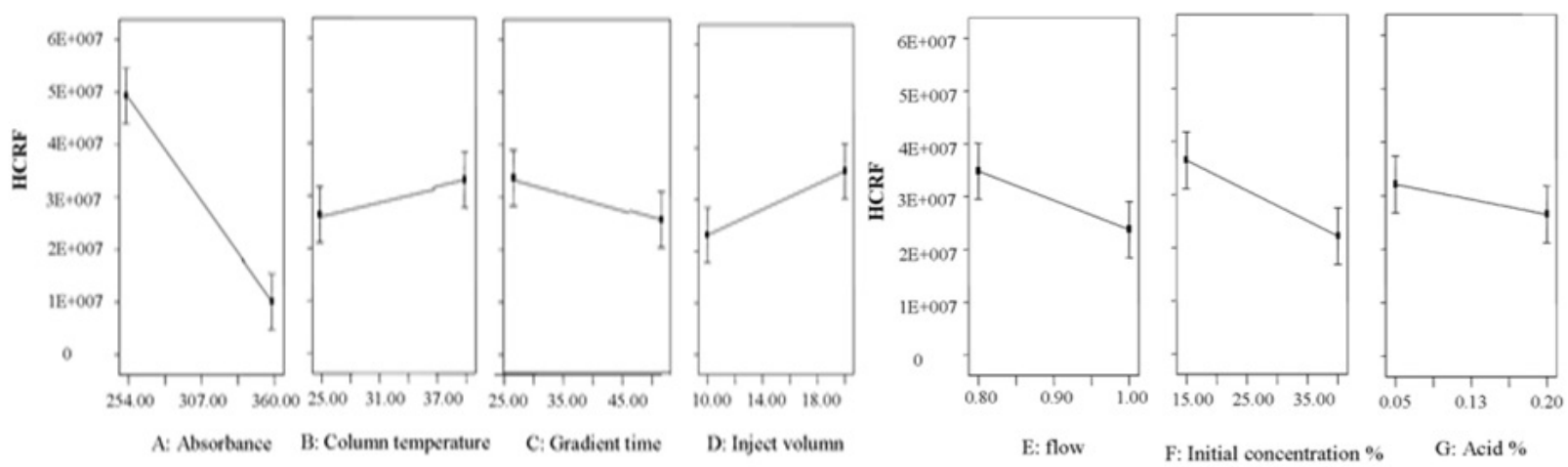

Figure 1: Response one-factor plots for main effect of HRCF, $254 \mathrm{~nm}$ wavelength measurement has distinctively effect on the HCRF.

\begin{tabular}{|l|l|l|l|}
\hline \\
(a)
\end{tabular}
Figure 2: a, b shows that the detection wavelength had better effects on
with increase in initial organic phase, the response surface decreases gra
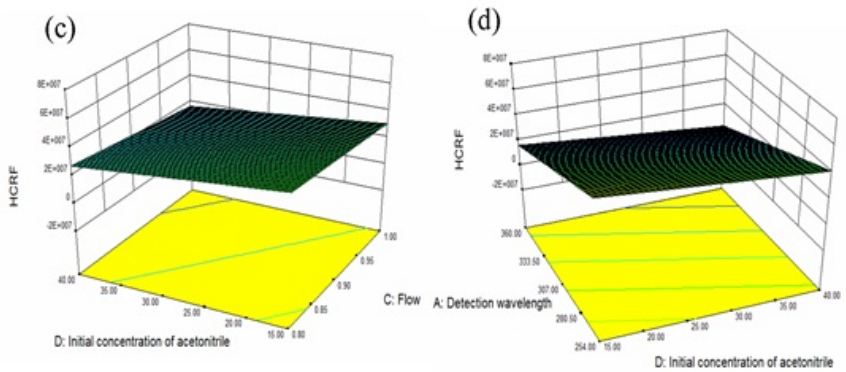

Figure 2: $\mathrm{a}, \mathrm{b}$ shows that the detection wavelength had better effects on the surface than the increasing sample volume, while $\mathrm{c}$, $\mathrm{d}$ shows that with increase in initial organic phase, the response surface decreases gradually, but the flow velocity has no effect on it.

\section{Fingerprint similarity evaluation} Chinese medicine (TCM) chromatographic fingerprint similarity evaluation system for the median similarity evaluation, the result shows that the average similarity was $96.0 \%$ (as seen table 2).

\begin{tabular}{|l|l|l|l|l|}
\hline Mean & & & & 0.96 \\
\hline
\end{tabular}

Table 2: HPLC fingerprint compared between Hypericum perforatum L. extracts and standard extracts.

\section{Method Validation}

\section{Peak identification}

According to the standard chromatogram separation in the same chromatographic conditions, Main active compounds peak were identified from the fingerprint (Figure 3). 
Page 4 of 5

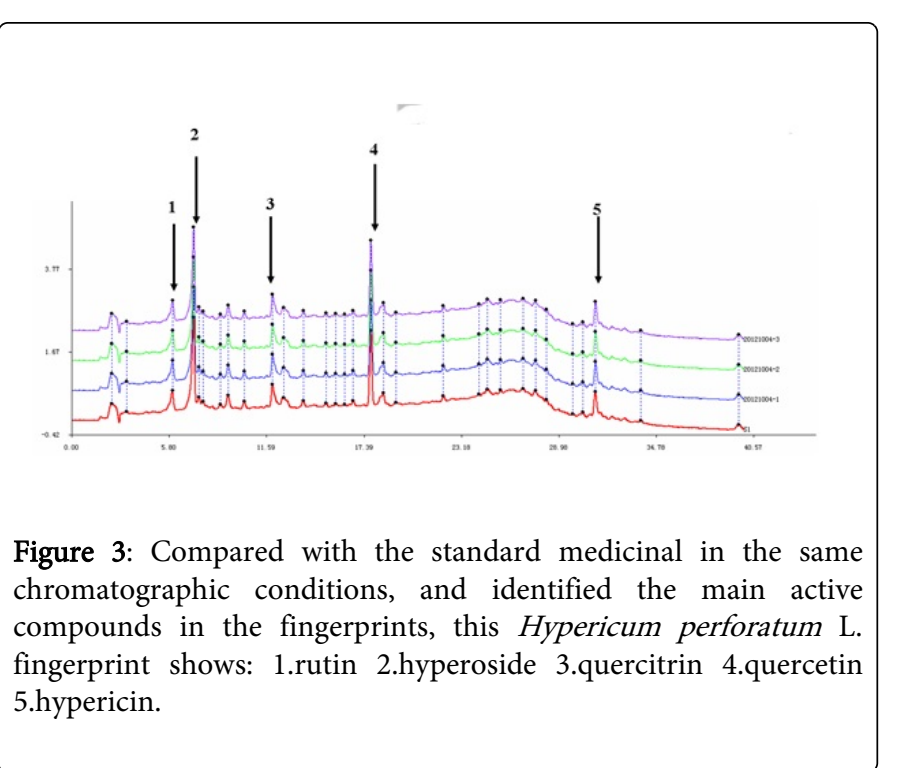

\section{Linearity and range}

Hyperoside standards sample (5 mg) was weighed accurately into a $25 \mathrm{ml}$ brown volumetric flask. Added the mobile phase to the scale line, the stock solution was made to standard solution. Used the mobile phase concentration to dilution and $0.22 \mu \mathrm{m}$ membrane to filter, the standard stock solution was mixed into $10 \sim 100 \mu \mathrm{g} / \mathrm{ml}$ linear working fluid. Besides, the working fluid was transferred to the chromatograms with $20 \mu \mathrm{l}$ sample injection. After the test, calculation of the equation was $\mathrm{Y}=37393 \mathrm{X}+71352, \mathrm{r}=0.9995$ (Figure 4).

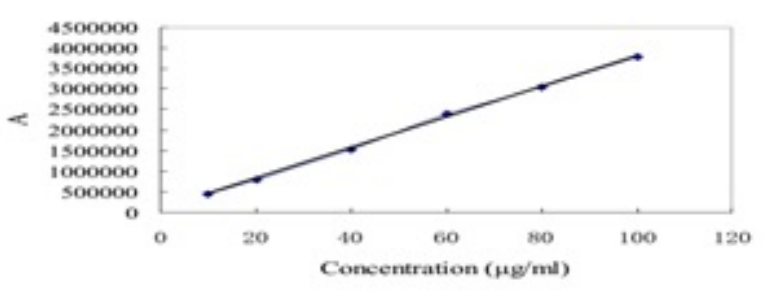

Figure 4: Linearity and range standard curves of hyperoside $(10 \sim 100 \mu \mathrm{g} / \mathrm{ml})$. It reveals hyperoside had better linearity in range from $10 \mu \mathrm{g} / \mathrm{ml}$ to $100 \mu \mathrm{g} / \mathrm{ml}(50 \% \sim 500 \% \mathrm{LC})$.

\section{Precision test}

The standard stock solution of hyperin $(20 \mu \mathrm{g} / \mathrm{ml})$ were diluted with the mobile phase to prepare the concentration $80 \%, 100 \%$ and $120 \%$, respectively. Each concentration had three copies and used $0.22 \mu \mathrm{m}$ membrane to filter. Besides, the working fluid was transferred to the chromatograms with $20 \mu \mathrm{l}$ sample injection. Calculating the method of precision and the inter day precision for each concentration. It shows that $80 \%, 100 \%$ and $120 \%$ solution precision were $0.52 \%, 0.36 \%$ and $1.28 \%$. Meanwhile, the $72 \mathrm{~h}$ solution between day precision were $0.62 \%, \quad 0.45 \%$ and $0.16 \%$. Our study which established chromatographic conditions about the precision method and the inter day precision were successful.

\section{Recovery test}

According to Chinese pharmacopoeia [6] of traditional Chinese medicine (TCM) in the provisions of the quality control methods (The pharmacopoeia of the People's Republic of China. 2010), Hypericum perforatum L. extract powder of hyperoside (1 g, 6 copies) were weighed accurately into $10 \mathrm{ml}$ brown volumetric flasks. Hyperoside standard stock solution $(1 \mathrm{ml})$ was added to each copy and used the mobile phase concentration to diluting. Dissolved by the ultrasonic and using $0.22 \mu \mathrm{m}$ membrane to filter, the solution was transferred to the chromatograms with $20 \mu \mathrm{l}$ sample injection. Finally, the rate of hyperin recovery was $98 \sim 102 \%, \mathrm{RSD}=1.69 \%$, which illustrated this method had got a better accuracy

\section{Determination of the content}

Hypericum perforatum L. extract powder $(5 \mathrm{~g})$ were weighed accurately into the $25 \mathrm{ml}$ brown volumetric flask. Besides, mobile phase was added to the scale line. Solutions which parallel to prepare three copies were dissolved by ultrasonic about $30 \mathrm{~min}$ and used 0.22 $\mu \mathrm{m}$ membrane to filter. What's more, the solution was transferred to the chromatograms with $20 \mu \mathrm{l}$ sample injection. Finally, according to the external standard method, Hypericum perforatum L. extract powder of hyperoside was calculated by the peak area. The content of hyperoside was $0.026 \%, \mathrm{RSD}=0.67 \%$.

\section{Conclusion}

Plackett-Burman design proves to be a powerful tool for optimizing variables. The study shows $254 \mathrm{~nm}$ wavelength measurement had significant influence on it, and had afford better optimized parameters from One-factor plots for main effect of HCRF. In conclusion, Plackett-Burman (PB) design of experiment have established the appropriate methods to separate and to identify about flavonoids on the alcohol extract of Hypericum perforatum L., which could be widely used in quality control of compound extract and quality assessment process.

\section{Acknowledgment}

This work was supported by the National Natural Science Foundation of China (Project No. 21375147), Beijing Natural Science Foundation (Project No. 7142125) and the National Key Technologies R\&D Program for New Drugs of China (No. 2012ZX09301003-001-010).

\section{References}

1. Amara AA, Shibl AM, Tawfik AF (2012) Deeper in the antibiotics paradigm: microbes react individually and collectively for spreading the resistance. Afr J Microbiol Res 6: 2010-2019.

2. Amara AA (2011) Experimental design for simultaneous production of PHB, proteases and lipases. IIUM Eng J, 155-184.

3. Plackett RL, Burman JP (1946) The design of optimum multifactorial experiments. Biometrika 37: 305-325.

4. Yanru Liu, Rongqing Huang, Junxing Dong, Chenggang Zhang (2014) ABTS - + Scavenging Potency of Selected Flavonols from Hypericum perforatum L. by HPLC-ESI/MS QQQ: Reaction Observation, Adduct Characterization and Scavenging Activity Determination. Applied Food Research International, 58: 47-58.

5. Li S, Liu X, Zhu Y, Dong F, Xu J, et al. (2014) A statistical approach to determine fluxapyroxad and its three metabolites in soils, sediment and sludge based on a combination of chemometric tools and a modified 
Citation: Shen S, Liu YR, Guo CH, Huang RQ, Yang JY, et al. (2014) Optimization and Validation of a Fingerprint about Hypericum perforatum L. Extracts by Plackett-Burman Randomization Method. J Cytol Histol 6: 301. doi:10.4172/2157-7099.1000301

Page 5 of 5

quick, easy, cheap, effective, rugged and safe method. J Chromatogr A 1358: 46-51.
6. The pharmacopoeia of the People's Republic of China (2010) ISBN 1 97-7-506-7-9-443 A national pharmacopoeia committee. Beijing, PR china. 